\title{
Parotid Adenoid Cystic Carcinoma: A Case Report and Review of the Literature
}

Sepúlveda Ilson ${ }^{1 *}$, Frelinghuysen Michael$^{2}$, Platín Enrique ${ }^{3}$, Ortega Pablo ${ }^{4}$ and Delgado Carolina ${ }^{5}$

${ }^{1}$ Maxillofacial-Head and Neck Radiologist, ENT-Head and Neck Surgery Service, General Hospital of Concepcion, Chile ${ }^{2}$ Physician, Radiation Oncologist, Oncology Service, General Hospital of Concepcion, Chile

${ }^{3}$ Professor of Oral and Maxillofacial Radiology, University of North Carolina School of Dentistry, Chapel Hill, NC, USA

${ }^{4}$ Physician, Otolaryngologist, ENT-Head and Neck Surgery Service, General Hospital of Concepcion, Chile

5Physician Pathologist, Pathology Department, General Hospital of Concepción, University of Concepcion School of Medicine, Concepcion, Chile

\begin{abstract}
We report on a patient who presented to the ENT service with swelling of the right side of the parotid gland. The swelling had been present for four years. Imaging studies revealed an expansive process confined to the superficial right parotid lobule. The affected area was well delineated with irregular enhancement post intravenous contrast media administration. Surgical biopsy concluded the presence of Adenoid Cystic Carcinoma. The patient was treated with adjuvant radiation therapy and follow up exams confirm there is no evidence of recurrence.
\end{abstract}

\section{Introduction}

Adenoid cystic carcinoma (ACC) is malignant epithelial tumors that most commonly occur between the 5th and 6th decades of life. It is a slowly growing but highly invasive cancer with a high recurrence rate. This tumor has the propensity for perineural invasion. CT and Magnetic Resonance Imaging (MRI) studies with and without intravenous contrast media are commonly used to determine margins, extension, tumor infiltration pattern, as well as the presence of at the base of the skull.

\section{Clinic Case}

We report on a 74 years old female patient who presented to the ENT service complaining of right parotid swelling of four year duration. Palpation of the area revealed a mass approximately 6 centimeters in diameter with

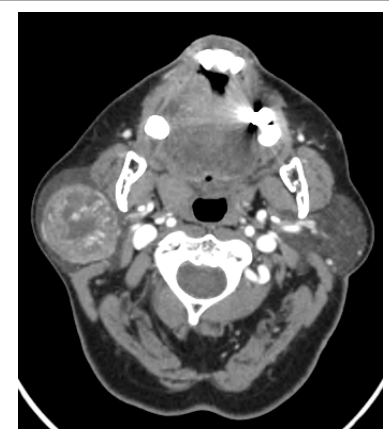

Figure 1: Expansive process in right superficial parotid lobule.

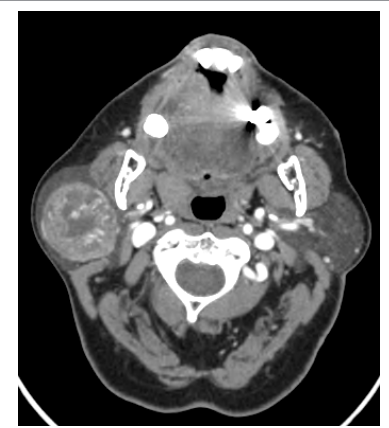

Figure 2: Heterogeneous mass showing central necrosis accompanied by irregular enhancement.

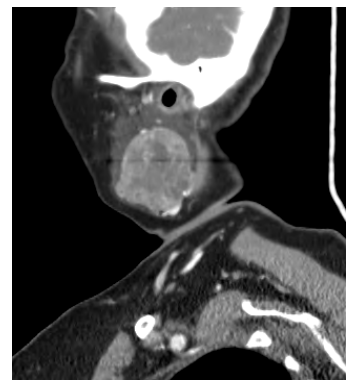

Figure 3: The mass encompassed the sternocleidomastoid muscle.

absence of pain. Her medical history indicated that she is currently taking medication for Arterial Hypertension and Diabetes Mellitus.

A Computed Tomography (CT) exam revealed an oval hypodense, heterogeneous expansive process confined to the superficial right parotid lobule (Figure 1). The lesion was well delineated with small calcification in the periphery. The lesion measured 4.7 centimeters in diameter at its widest point and post intravenous contrast media administration showed irregular enhancement (Figure 2). The mass encompassed the sternocleidomastoid and masseter muscles (Figure 3). Non-specific lymph nodes of 1 centimeter in diameter in IB and IIA levels were observed. Pharyngeal mucosal and parapharyngeal spaces were intact with no evidence of anatomical changes.

Surgical treatment with suprafacial parotidectomy was performed revealing a well-defined, encapsulated, non-infiltrating tumor. The post-surgical biopsy showed in H\&E stain, a classic cribiform

*Corresponding author: Sepúlveda Ilson, Maxillofacial-Head and Neck Radiologist, ENT-Head and Neck Surgery Service, General Hospital of Concepcion, Chile, Tel: +3531630 1301; Fax: +353 1630 1306; E-mail: isepulvedaguilar@gmail.com

Received: September 25, 2015; Accepted: October 13, 2015; Published: October 16,2015

Citation: Ilson S, Michael F, Enrique P, Pablo O, Carolina D (2015) Parotid Adenoid Cystic Carcinoma: A Case Report and Review of the Literature. Oncol Cancer Case Rep 1:101.

Copyright: (c) 2015 Ilson S, et al. This is an open-access article distributed under the terms of the Creative Commons Attribution License, which permits unrestricted use, distribution, and reproduction in any medium, provided the original author and source are credited. 
Citation: Ilson S, Michael F, Enrique P, Pablo O, Carolina D (2015) Parotid Adenoid Cystic Carcinoma: A Case Report and Review of the Literature. Oncol Cancer Case Rep 1:101.

Page 2 of 3

pattern and epithelial cells nests that form cylindric patterns. Inmunohistochemestry study was positive for Cytokeratin stain, resulted in a diagnosis of Adenoid Cystic Carcinoma with positive lymph involvement (Figure 4). Based on the size of the tumor the Head and Neck Cancer Committee recommended adjuvant radiotherapy (Figure 5). Follow up exams indicate the patients remains disease free (Figure 6).

\section{Discussion}

Adenoid cystic carcinoma (ACC) is a rare epithelial tumor comprising about $1 \%$ of all malignant tumors of the oral and maxillofacial region [1]. ACC is commonly grouped with salivary gland tumors, even though they may arise in sites where mucous glands exist. Half of these tumors occur in glandular areas other than the major salivary glands. They can also occur in the tongue, uvula and sites of minor salivary glands $[2,3]$.

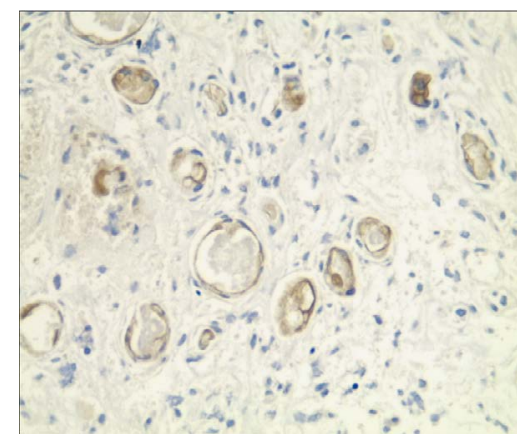

Figure 4: A. Hemotoxylin \& eosin stain; B. Positive Cytokeratin stain.

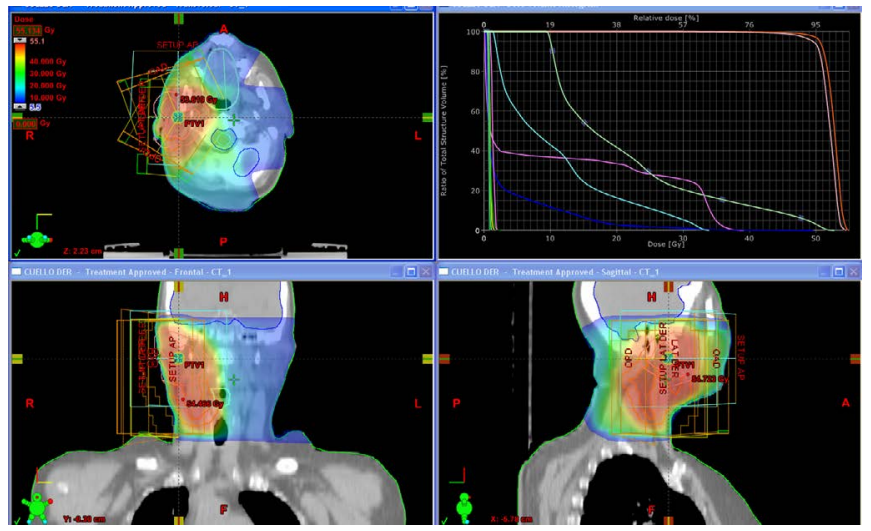

Figure 5: Dimentional Conformal Radiotherapy plan.

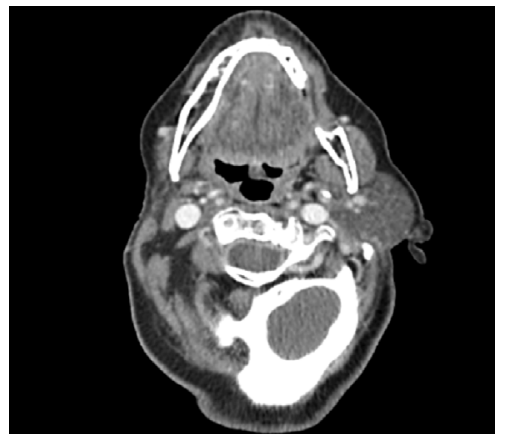

Figure 6: Follow up CT after 6 months: patient appears to be disease free.
ACC tumors present a widespread age distribution, the peak incidence occurs predominantly among women, between the 5th and 6th decades of life [4]. It is a slowly growing but highly invasive cancer with a high recurrence rate. This tumor has the propensity for perineural invasion; the tumor tissue often can extend far beyond the tumor's margin. Unfortunately, the tumor's slow growth may delay diagnosis for several years, allowing advanced perineural invasion (PNI) at the time of surgery. PNI spread has long been established. The literature has shown that the region of Gasserian ganglion is the most common site of involvement (35.8\%) [5].

PNI is grouped into two categories: incidental and clinical. Incidental PNI includes asymptomatic patients with evidence of microscopic PNI detected only by histopathology. Clinical PNI includes patients with evidence of a cranial neuropathy upon physical examination and/or radiographic evidence of gross tumor involvement along the tract of the nerve. Magnetic resonance imaging (MRI) is used to detect and define the extent of PNI. It is important to note, it is rare to observe radiographic evidence of PNI in the asymptomatic patient. PNI is sometimes associated with "skip" lesions along the nerve, which significantly increases the risk of recurrence after surgical resection even if negative margins are obtained [6]

Lymphatic spread to local lymph nodes is rare. Hematogenous spread occurs often in the course of the disease [7]. Metastases to the lung is more common than regional lymph node metastasis [8]. The clinical differential diagnosis for such palatal swelling may include salivary gland neoplasms (such as pleomorphic adenoma, myoepithelioma, mucoepidermoid carcinoma, adenocarcinoma), necrotizing sialometaplasia, odontogenic tumors such as ameloblastoma, calcifying epithelial odontogenic tumor, odontogenic myxoma, clear cell odontogenic tumor, odontogenic carcinoma and hematopoietic neoplasm such as non-Hodgkin's lymphoma [9].

Histopathologically, ACC presents in three patterns; cribriform, tubular and solid. The cribriform glandular pattern is the most common and best recognized appearance. It is characterized by islands of basaloid epithelial cells that contain multiple cylindrical areas $[9,10]$. Histologically, this entity has been classified in three subgroups; Grade I defined as well differentiated and composed of tubular and cribriform patterns without solid components; grade II characterized by a pure cribriform pattern or mixed with less than $30 \%$ of solid areas; and grade III predominantly solid in pattern $[11,12]$.

CT and Magnetic Resonance Imaging (MRI) studies with and without intravenous contrast media are commonly used to determine margins, extension, tumor infiltration pattern, as well as the presence of at the base of the skull. Metastasis and extension into various structures are generally evaluated by means of radiographic images, ultrasound imaging and scintillography. Admittedly, imaging exams are key modalities used as diagnostic and post treatment follow up procedures [13].

The incidence of clinically positive neck nodes at diagnosis is relatively low [14]. The management of these types of tumors involves a multidisciplinary approach and surgical resection is the treatment of choice. Due to the rarity of ACC, randomized trials of postoperative radiotherapy (PORT) patients have not been conducted, however; several retrospective studies have shown improved outcomes in patients treated with PORT. Many retrospective studies have also included patients treated with radiation therapy alone for inoperable cancers $[14,15]$

PORT is also indicated for certain tumor locations. For example, those close to the base of the cranium with the presence of neck lymph 
Citation: Ilson S, Michael F, Enrique P, Pablo O, Carolina D (2015) Parotid Adenoid Cystic Carcinoma: A Case Report and Review of the Literature. Oncol Cancer Case Rep 1:101.

node metastasis and PNI, the solid histological subtype, and tumors that have recurred. Several authors, on the other hand doubt that postoperative radiation may influence the course of the disease [16]. Also, other suggest that only surgical removal with clean surgical margins or radiotherapy will reduce the possibility of recurrence and metastasis in cervical lymph nodes, lungs, bones and brain [17].

Chen et al. [14] reported on 140 patients treated with definitive surgery with and without postoperative radiotherapy. They concluded that combined-modality therapy with surgery followed by radiotherapy doses in excess of $60 \mathrm{~Gy}$ should be considered the standard of care for adenoid cystic carcinoma of the head and neck. Also, Garden et al. [18] reported on 198 patients treated with surgery and postoperative radiotherapy at the MD Anderson Cancer Center (Houston, Tex) between 1962 and 1991. The rates of freedom from relapse and overall survival, rates were $68 \%$ and $82 \%$ at 5 years, $52 \%$ and $65 \%$ at 10 years, and $45 \%$ and $48 \%$ at 15 years.

Although the combination of concomitant chemotherapy and radiotherapy is more efficacious than radiotherapy alone for patients with advanced squamous cell carcinomas of the head and neck [19], experience with adjuvant chemotherapy for patients with ACCs is limited. It is tempting to add adjuvant chemotherapy to Radiotherapy alone or combine it with surgery because of the high risk of hematogenous dissemination following treatment; however, there is no convincing evidence that adjuvant chemotherapy is beneficial for patients with ACC $[20,21]$.

A variety of prognostic factors have been studied and established; including the presence of cervical lymphatic metastases at the time of diagnosis, advanced tumor stage, the presence of affected surgical margins, solid histological type or histopathology grade and macroscopic PNI $[18,22,23]$. Tumor free surgical margins can be correlated with better local control and also with longer survival rateS [18]. Sur et al. [24], noted that although the extension of the surgical resection has a significant impact on local control and on disease-free survival, it fails to make an impact on overall survival due to the slow growth that characterizes these tumors.

\section{Conclusion}

Adenoid Cystic Carcinoma is a rare entity, with malignant epithelial components and propensity for PNI. Imaging studies play an important role in the diagnosis and treatment planning. These exams provide valuable information about the growing pattern and involvement of critical anatomic organs. The treatment of choice is surgical resection, combined with adjuvant radiotherapy in some cases.

\section{References}

1. Kokemueller H, Eckardt A, Brachvogel P, Hausamen JE (2004) Adenoid cystic carcinoma of the head and neck - a 20 years experience. Int J Oral Maxillofac Surg 33: 25-31.

2. Berdal $P$ (1970) Cylindroma of salivary glands: a report of 80 cases. Acta Otolaryngol 263: 170-173.

3. Spiro RH (1986). Salivary neoplasms: overview of a 35-year experience with 2 , 807 patients. Head Neck Surg 8: 177-184.
4. Waldron CA, El-Mofty SK, Gnepp DR (1988) Tumors of the intraoral minor salivary glands: a demographic and histologic study of 426 cases. Oral Surg Oral Med Oral Pathol 66: 323-333.

5. Wakisaka S, Nonaka A, Morita Y, Fukui M, Kinoshita K (1990) Adenoid cystic carcinoma with intracranial extension: report of three cases. Neurosurgery 26 1060-1065.

6. Balamucki C J (2012) American Journal of Otolaryngology-Head and Neck Medicine and Surgery 33: 510-518.

7. Huang MX, MaD, SunK, YuG, GuoC, et al. (1997) Factors influencing survival rate in adenoid cystic carcinoma of the salivary glands. Int $\mathrm{J}$ Oral Maxillofac Surg 26: 435-439.

8. Neville BW, Damm DD, Allen CM, Bouquot JF (2002) Salivary gland pathology, Oral \& maxillofacial pathology. W.B. Saunders Co, Philadelphia, USA

9. Gondivkar SM (2011) Adenoid Cystic Carcinoma: A rare clinical entity and literature review. Oral Oncology 47: 231-236.

10. Batsakis JG, Luna MA (1990) Histopathologic grading of salivary gland neoplasms: III. Adenoid cystic carcinomas. Ann Otol Rhinol Laryngol 99: 10071009.

11. Szanto PA, Luna MA, Tortoledo ME, White RA (1984) Histologic grading of adenoid cystic carcinoma of the salivary glands. Cancer 54: 1062-1069.

12. Chen JC, Gnepp DR, Bedrossian CW (1988) Adenoid cystic carcinoma of the salivary glands: an immunohistochemical analysis. Oral Surgery Oral Medicine Oral Pathol 65: 316-326.

13. Lupinetti AD, Roberts DB, Williams MD, Kupferman ME, Rosenthal DI, et al (2007) Sinonasal adenoid cystic carcinoma: the M. D. Anderson Cancer Center experience. Cancer 110: 2726-2731.

14. Chen AM, Bucci MK, Weinberg V, Garcia J, Quivey JM et al. (2006) Adenoid cystic carcinoma of the head and neck treated by surgery with or without postoperative radiation therapy: prognostic features of recurrence. Int J Radiat Oncol Biol Phys 66: 152-159.

15. Gomez DR, Hoppe BS, Wolden SL, Zhung JE, Patel SG, et al. (2008) Outcomes and prog-nostic variables in adenoid cystic carcinoma of the head and neck: a recent experience. Int J Radiat Oncol Biol Phys 70: 1365-1372.

16. Prokopakis EP, Snyderman CH, Hanna EY, Carrau RL, Johnston JT, et al. (1999) Risk factors for local recurrence of adenoid cystic carcinomas. Am J Otolaryngol 20: 281-286

17. Grisius MM, Fox PC (2003) Salivary gland diseases, Oral medicine diagnosis and treatment. BC Decker Inc, Hamilton, USA.

18. Garden AS, Weber RS, Morrison WH (1995) The influence of positive margins and nerve invasion in adenoid cystic carcinoma of the head and neck treated with surgery and radiation. Int J Radiat Oncol BiolPhys 32: 619-626.

19. Mendenhall WM, Riggs CE, Vaysberg M (2010) Altered fractionation and adjuvant chemotherapy for head and neck squamous cell carcinoma. Head Neck 32: 939-945.

20. Diaz Jr EM, Kies MS (2001) Chemotherapy for skull base cancers. Otolaryngo Clin North Am 34: 1079-1085.

21. Vincentelli F, Grisoli F, Leclercq TA, Ardaud B, Diaz-Vasquez P, et al. (1986) Cylindromas of the base of the skull. J Neurosurg 65: 856-859.

22. Fordice J, Kershaw C, El-Naggar A, Goepfert H (1999) Adenoid cystic carcinoma of the head and neck: predictors of morbidity and mortality. Arch Otolaryngol Head Neck Surg 125: 149-152.

23. Spiro RH, Hooves AG, Strong EW (1979) Adenoid cystic carcinoma: factors influencing survival. Am J Surg 138: 579-583.

24. Sur RK, Donde B, Levin V, Pacella J, Kotzen J, et al. (1997) Adenoid cystic carcinoma of the salivary glands: a review of 10 years. Laryngoscope 107 1276-1280. 